\title{
6-芳酰基菲啶合成反应的机理及取代基电子 效应研究
}

\author{
徐婷，戚孝天，蓝宇*
}

重庆大学化学化工学院, 重庆 400030

*通讯作者, E-mail: lanyu@cqu.edu.cn

收稿日期: 2015-11-02; 接受日期: 2015-11-16; 网络版发表日期: 2016-03-09

国家自然科学基金(编号: 21372266, 51302327)、重庆大学“青年百人计划”(编号: 0903005203191)和中央高校(重庆大学)基础研究经费(编 号: 106112015CDJZR228806)资助项目

\begin{abstract}
摘要本文运用密度泛函理论(DFT)计算, 系统研究了碱促均裂芳香取代合成 6-酰基菲啶的反应机理. 计算结 果表明，叔丁氧自由基和苯甲醛间的自由基传递反应是整个反应的决速步骤. 随后酰基自由基对氯基的亲核进 攻以及自由基环化过程是快速步骤. 此外, 我们还利用 Hammett 曲线研究了反应过程中不同位置上取代基的电 子效应。研究结果表明，氯基对位上被吸电子基团取代时有利于酰基自由基的亲核进攻，酰基对位被给电子基 团取代时有助于自由基环化过程的发生.
\end{abstract}

关键词菲啶, 密度泛函理论计算, 自由基反应, Hammett 曲线, 取代基电子效应

\section{1 引言}

重要的含氮杂环菲啶是多种药物 ${ }^{[1 \sim 4]}$ 、天然产 物 ${ }^{[5 \sim 7]}$ 、和生物分子的核心结构 ${ }^{[8 \sim 10]}$. 菲啶类化合物具 有很多重要生物活性(抗菌、抗细胞毒素、抗癌等 $)^{[1114]}$ 和光电特性 ${ }^{[15]}$, 是现代有机合成和生物分子制备中 的重要前体. 鉴于菲啶结构单元的广泛用途, 如何经 济、高效地合成菲啶及其衍生物在医药化学和有机合 成领域中具有重要的意义.

目前对于菲啶结构单元的构建一般是通过邻取 代二芳基化合物的分子内环化实现的，具体合成方

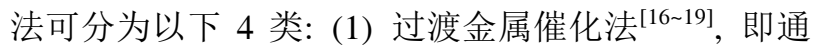
过过渡金属催化 C-N 键偶联构建菲啶; (2) 光化学环
化法 ${ }^{[20-22]}$, 即通过光照催化实现分子内的环化; (3) 多组分一锅串联法 ${ }^{[23 \sim 25]}$, 即通过一锅煮的方法由多

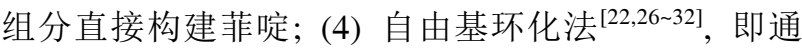
过自由基对芳环亲核进攻得到分子内环化的产物. 随着近段时间自由基化学的快速发展，通过自由基 环化法合成菲啶及其衍生物这一策略受到了越来越 来多的关注.

2012 年, Chatani 等 ${ }^{[27]}$ 报道了由 2-异氰基联苯和 芳基硼试剂的氧化环化反应制备菲啶. 其中芳基硼 试剂在氧化剂锰(III)的存在下会均裂产生芳基自由 基，芳基自由基进攻异氰末端碳，随后分子内的自由 基加成可以得到环化中间体. 此外, Studer 课题组 ${ }^{[28]}$ 和 Zhou 课题组 ${ }^{[29]}$ 分别报道了以 2-异氰基联苯作自由

引用格式: 徐婷, 戚孝天, 蓝宇. 6-芳酰基菲啶合成反应的机理及取代基电子效应研究. 中国科学: 化学, 2016, 46: 616-622

Xu T, Qi XT, Lan Y. Mechanism and electronic effect for the synthesis of 6-aroylated phenanthridines: a theoretical study. Sci Sin Chim, 2016, 46: 616-622, doi: 10.1360/N032015-00217 
基受体, 以三氟甲基自由基作引发剂, 经过自由基环 化过程合成 6-三氟甲基取代菲啶衍生物. 在此基础 上, Studer 课题组 ${ }^{[32]}$ 研究了通过均裂芳香取代得到 6芳酰基菲啶的合成方法. 如图 1 所示, 该反应用双芳 基异腈与苯甲醛结合作为碳自由基的前体, 以乙腈作 为溶剂, 过氧叔丁醇作为氧化剂, 氯化铁为自由基引 发剂, 通过自由基环化过程合成 6-芳酰基菲啶. 该反 应使用简单易得的底物和极少量的铁就可以制备 6-芳 酰基菲啶衍生物, 具有很大的应用前景. 尽管对于通 过自由基环化法构建菲啶结构单元的实验研究有很 多, 相应的理论研究却鲜有报道.

为了更好地理解这类反应, 我们使用密度泛函 理论(DFT)方法研究其反应机理以及苯环上取代基电 子效应对反应产率的影响. 计算以 Studer 课题组报道 的碱促均裂芳香取代制备 6-芳酰基菲啶的反应为模 板反应.

\section{2 计算方法}

本文中的计算均采用 GAUSSIAN09 量子化学程 序包 ${ }^{[33]}$ 完成. 密度泛函方法 $\mathrm{B} 3-\mathrm{LYP}^{[34-37]}$ 和基组 $6-31+\mathrm{G}(\mathrm{d}, \mathrm{p})$ 用来对反应势能曲线上的中间体和过渡态 进行结构优化. 简谐频率振动计算用来确认每一个驻 点是稳定的中间体或者过渡态, 同时也用来得到每一 个驻点焓和吉布斯自由能的校正值. 为了得到更加准 确的能量数值, 在气相驻点结构的基础上, 使用 M1 $1^{[38 \sim 41]}$ 方法和 $6-311+\mathrm{G}(\mathrm{d}, \mathrm{p})$ 基组来计算各驻点的溶 剂化单点能. 溶剂化计算时采用 SMD 连续溶剂模

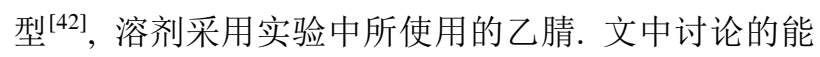
量值是 M11 计算得到的吉布斯自由能. 关键的反应中 间体和过渡态的几何构型由 CYLview 软件绘制.

\section{3 结果与讨论}

\section{1 自由基环化生成 6-芳酰基菲啶的反应机理}

结合实验条件、自由基反应的特性以及 Studer 课题组在文章中提出的机理, 对于碱促均裂芳香取 代合成 6-取代菲啶的反应, 我们首先提出了一条可 能的反应路径(图 2). 反应过程中, 叔丁基过氧化氢 (TBHP)在加热条件下均裂产生叔丁氧自由基和羟基 自由基, 羟基自由基在 $\mathrm{FeCl}_{3}$ 存在情况下被还原得到 羟基负离子. 叔丁氧自由基与反应底物苯甲醛发生

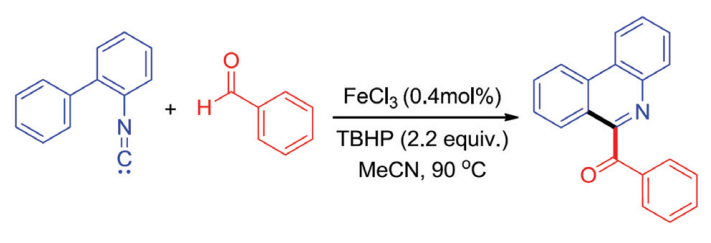

图 1 碱促均裂芳香取代合成 6-芳酰基菲啶

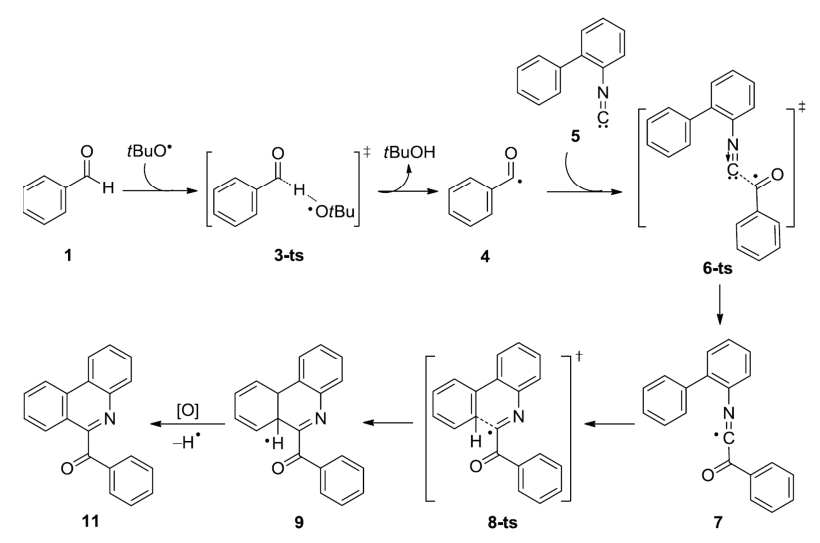

图 2 碱促均裂芳香取代合成 6-取代菲啶的反应途径

氢自由基转移反应, 经过渡态 3-ts 生成酰基自由基 4. 随后酰基自由基 4 进攻 2-异氧基联苯上末端碳原子, 由过渡态 6-ts 产生亚胺自由基中间体 7. 之后发生分 子内自由基环化, 经过一个六元过渡态 8-ts 生成环己 二烯基自由基 9. 最后中间体 9 在氧化剂作用下脱去 一分子氢自由基可以得到最终产物 6-芳酰基菲啶.

根据提出的反应路径, 我们首先计算了酰基自 由基的生成. 相应的势能曲线如图 3 所示, 叔丁氧自 由基与苯甲醛 1 被选作势能曲线的相对吉布斯自由 能零点. 通过叔丁氧自由基和苯甲醛羰基氢的分子间 氢键作用生成中间体 2 , 其相对自由能是 $7.8 \mathrm{kcal} / \mathrm{mol}$. 接着, 经过自由基传递过渡态 3-ts 可以生成酰基自由 基 4, 这一过程的活化能是 $17.1 \mathrm{cal} / \mathrm{mol}$. 过渡态 3-ts 的 CYLview 结构表明, 在形成过渡态时 $\mathrm{C}-\mathrm{H}$ 键的键 长是 $1.25 \AA$, $\mathrm{O}-\mathrm{H}$ 键的键长是 $1.34 \AA$. 另外, 酰基自 由基的相对自由能是 $-15.6 \mathrm{cal} / \mathrm{mol}$, 说明酰基自由基 4 的形成是放热过程.

生成的酰基自由基 4 会对 2-异氧基联苯亲核进 攻, 进攻位点是氰基末端碳原子. 由过渡态 6-ts 生成 亚胺自由基 7 的活化能是 $24.5 \mathrm{kcal} / \mathrm{mol}$. 亚胺自由基 7 的相对自由能是 $-25.6 \mathrm{cal} / \mathrm{mol}$ (图 4). 优化好的几何 结构表明(图 5), 在亲核进攻过渡态 6-ts 中 $\mathrm{C}-\mathrm{C}$ 键的 距离是 $2.25 \AA$, 异㲵末端 $\mathrm{C}-\mathrm{N}$ 键距离是 $1.19 \AA$. 另外, 


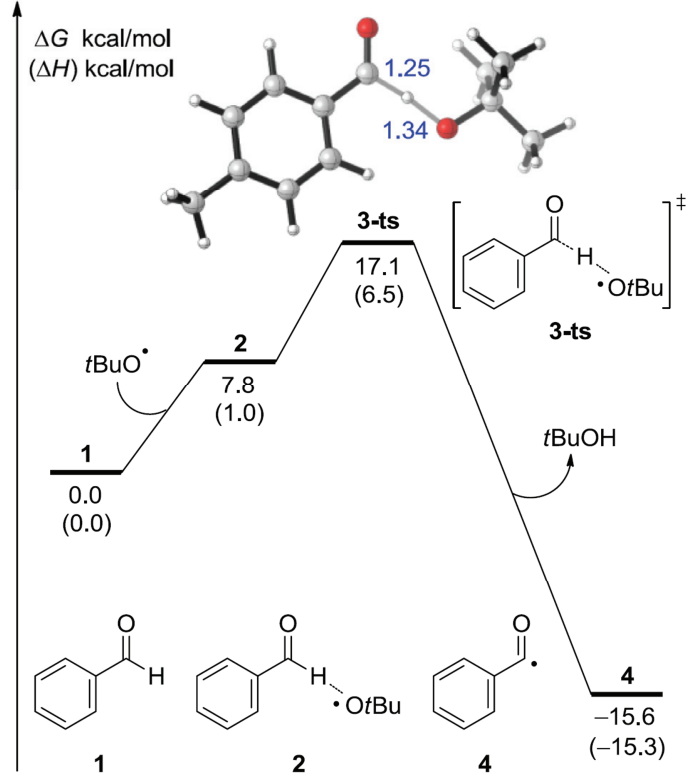

图 3 酰基自由基的生成(网络版彩图)

亲核进攻时 $\mathrm{C}-\mathrm{C}-\mathrm{N}$ 的夹角是 $109.8^{\circ}$, 这一数值非常 接近甲烷分子中碳氢键的夹角 $109.5^{\circ}$, 此时 $\mathrm{C}$ 的轨道 近似于 $\mathrm{sp}^{3}$ 杂化. 而在亚胺自由基 7 中, 相应的 $\mathrm{C}-\mathrm{C}$ 键的键长是 $1.41 \AA, \mathrm{C}-\mathrm{N}$ 键键长是 $1.18 \AA, \mathrm{C}-\mathrm{C}-\mathrm{N}$ 的 夹角是 $172.3^{\circ}$, 这说明中间体 7 中 $\mathrm{C}-\mathrm{C}-\mathrm{N}$ 的构型比 较接近直线型.

随后，在中间体 7 中会发生分子内自由基环化, 亚胺自由基进攻相邻的芳环, 由过渡态 8-ts 生成环化 中间体 9, 这一过程的活化能为 $14.5 \mathrm{kcal} / \mathrm{mol}$. 过渡 态 8-ts 的 CYLview 结构表明, 自由基环化过程中将 要形成的 $\mathrm{C}-\mathrm{C}$ 键键长是 $2.10 \AA$. 该过程中 $\mathrm{C}-\mathrm{N}$ 键的 键长是 $1.25 \AA$, 比中间体 7 中 $\mathrm{C}-\mathrm{N}$ 键的键长增长了 $0.07 \AA$. 而在形成中间体 9 后, 相应 $\mathrm{C}-\mathrm{C}$ 键的键长变 为 $1.53 \AA, \mathrm{C}-\mathrm{N}$ 键变为 $1.29 \AA$. 中间体 9 的相对自由 能是 $-39.5 \mathrm{kcal} / \mathrm{mol}$ (图 4), 这说明环化中间体 9 的生 成是不可逆过程.

最后，由羟基自由基与铁作用生成的羟基负离 子会进攻环己二烯基自由基中间体 9, 拔去 1 个质子 生成水分子，同时得到联芳基阴离子自由基 10. 紧 接着联芳基阴离子自由基 10 被过氧叔丁醇进一步氧 化失去 1 个电子, 生成 6-芳酰基菲啶产物 11. 分析整 个反应势能曲线, 酰基自由基进攻氰基过程和自由 基环化过程的活化能一致(14.5 kcal/mol), 而酰基自 由基生成的活化能是 $17.1 \mathrm{kcal} / \mathrm{mol}$, 所以反应的决速

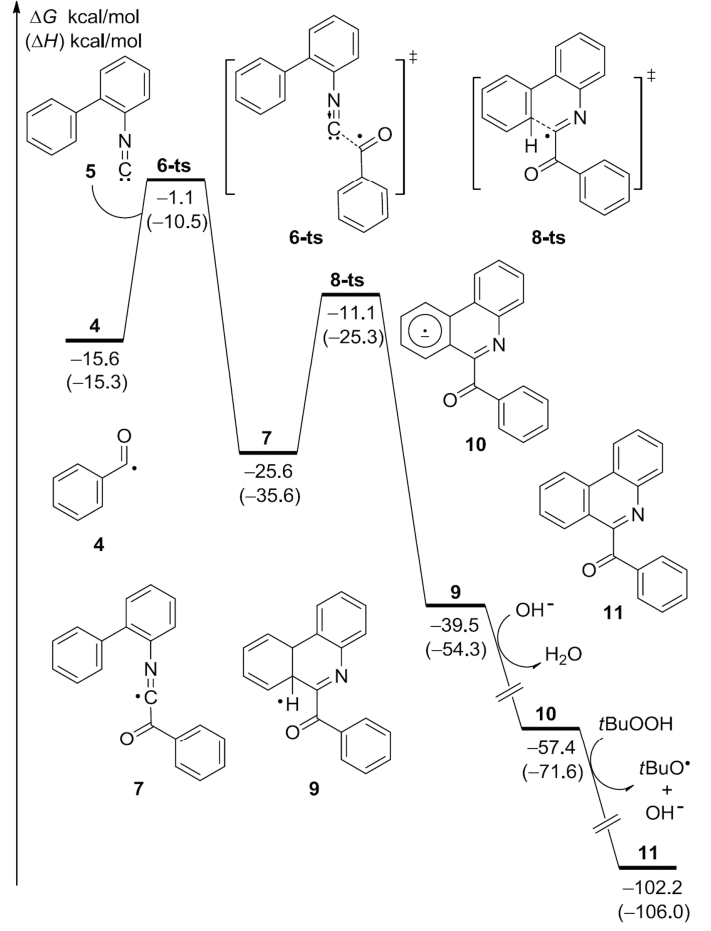

图 4 通过自由基环化生成 6-芳酰基菲啶的势能曲线
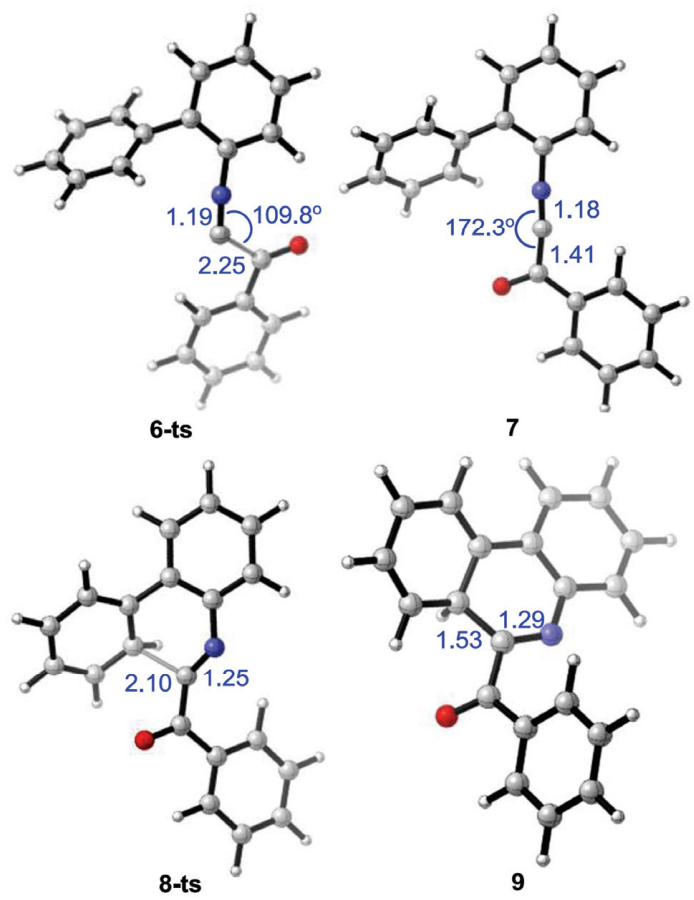

图 5 优化好的 6-ts、7、8-ts 和 9 的几何构型(网络版彩图)

步骤是酰基自由基的生成. 另外，6-芳酰基菲啶产物 11 的生成放热 $102.2 \mathrm{kcal} / \mathrm{mol}$, 说明反应具有很强的 
驱动力. 因此, 这个机理可以清楚合理地阐明反应路 径, 与实验现象一致.

\section{2 取代基电子效应对反应的影响}

经过理论计算, 我们确定了碱促均裂芳香取代 合成 6-芳酰基菲啶的反应机理. 通过模拟含有不同 取代基团的底物进行反应, 探讨底物上不同位置的 不同取代基团电子效应对酰基自由基进攻氧基过程 和自由基环化过程的影响. 对于取代基团, 除了氢, 我们选取了 3 个给电子基团 (二甲胺基 $\left(-\mathrm{NMe}_{2}\right)$ 、甲氧 基 $(-\mathrm{OMe}$ ) 和甲基 $(-\mathrm{Me})$ ), 以及 3 个吸电子基团(氯 $(-\mathrm{Cl})$ 、三氟甲基 $\left(-\mathrm{CF}_{3}\right)$ 和硝基 $\left(-\mathrm{NO}_{2}\right)$ ).

首先研究酰基自由基进攻氰基过程中取代基的 电子效应. 分别设计 2 -氭基联苯中氰基的对位上被 不同取代基取代的 2-氧基联苯衍生物与苯甲酰基自 由基反应, 苯环的对位被不同取代基的 2-氰基联苯 衍生物与苯甲酰基自由基反应, 以及对位被不同取 代基取代的苯甲酰基自由基与 2-㲵基联苯的反应. 使用同样的算法和基组分别计算这 3 个反应的过渡 态结构和能量, 绘制 Hammett 曲线 ${ }^{[43,44]}$, 以此研究取 代基团电子效应对酰基进攻异腈过程的影响.

在之前优化的过渡态结构基础上, 分别优化设 计的 3 个反应的中间体和过渡态结构, 整理酰基自由 基进攻氧基过程的活化能数值. 如图 6 所示, 以各取 代基的取代基常数 $\sigma$ 为横坐标, 以 $\log k_{\mathrm{X}} / k_{\mathrm{H}}$ 为纵坐标, 根据取代基所在位置的不同分别绘制 Hammett 曲线.

\subsection{1 酰基自由基进攻氰基过程取代基电子效应}

分析 Hammett 曲线, 当取代基在氧基对位时, 所 得的回归直线如图 6(a) 所示, 回归直线的拟合优度 $R^{2}$ 为 0.89 , 表明直线的拟合性很好, 数据具有很好的线 性关系. 另外, 回归直线的斜率为 0.32 , 即反应常数 $\rho>0$, 取代基常数 $\sigma$ 数值越大, 反应速率越快, 所以氭 基对位上连有吸电子基团(硝基、三氟甲基、氯原子) 时有利于酰基自由基对氧基的进攻, 而氧基对位上 连有给电子基团(甲基、甲氧基、二甲基胺基)时不利 于酰基自由基的进攻. 其原因是吸电子基团的存在, 使得氧基上的电荷密度降低, 有利于酰基自由基对 氧基的亲核进攻, 从而降低过渡态活化能.

当取代基在苯环的对位上时，得到的回归直线 如图 6(b)所示, 回归直线的拟合优度 $R^{2}$ 为 0.0013 , 且 直线的斜率接近于 0 . 回归直线的拟合性非常差, 说
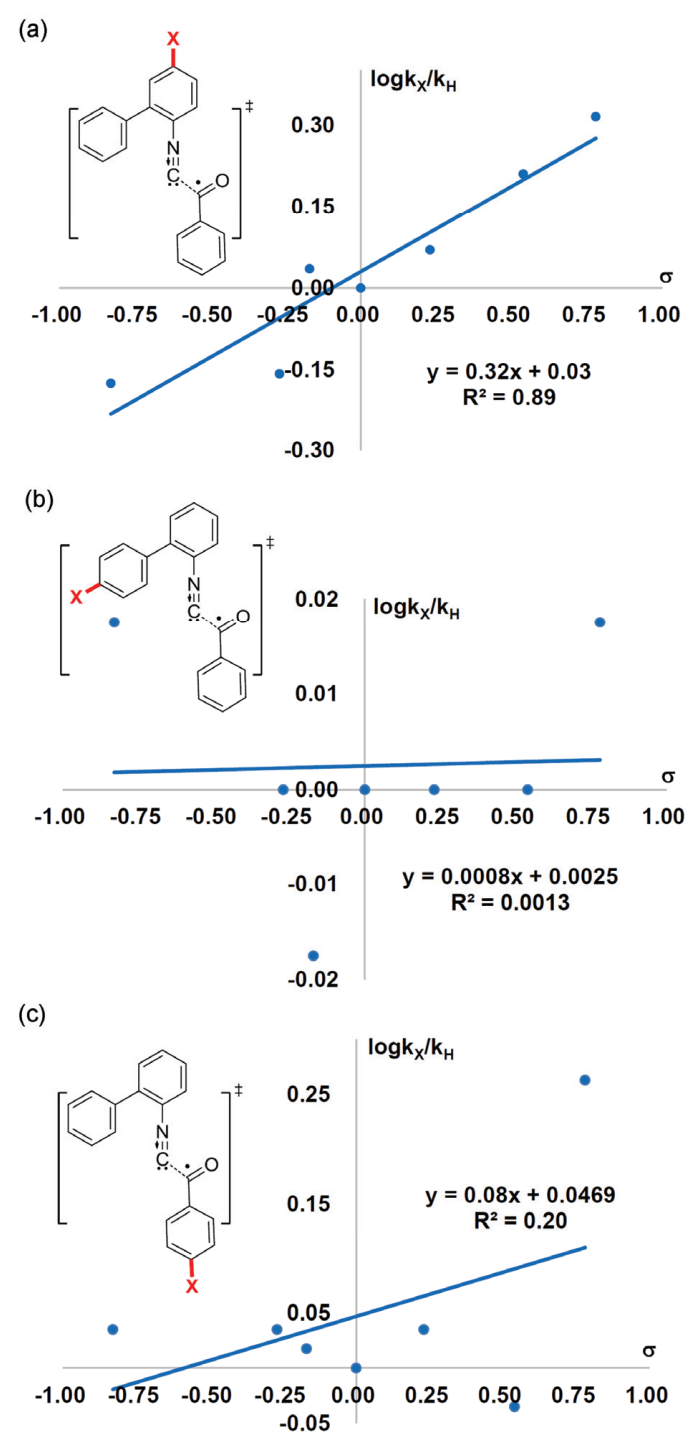

图 6 酰基自由基进攻氧基时不同取代位置的 Hammett 曲线. （a）取代基-X在氧基对位; (b) 取代基-X在苯环对位; (c) 取 代基-X 在酰基对位. 其中取代基 $-\mathrm{X}$ 分别为: $-\mathrm{NMe}_{2}(\sigma=$ $-0.83) 、-\mathrm{OMe}(\sigma=-0.27) 、-\mathrm{Me}(\sigma=-0.17) 、-\mathrm{H}(\sigma=0) 、-\mathrm{Cl}$ $(\sigma=0.23) 、-\mathrm{CF}_{3}(\sigma=0.54) 、-\mathrm{NO}_{2}(\sigma=0.78)$

明这组数据不具有线性相关性. 因此, 苯环对位上的 取代基电子效应对酰基自由基的亲核进攻速率基本 没有影响.

当取代基在酰基对位时, 得到的回归直线如图 6 (c)所示, 回归直线的拟合优度 $R^{2}$ 为 0.20 , 表明回归 直线的拟合性较差. 因此酰基对位上的取代基电子 效应对酰基进攻氰基过程的影响较小, 导致该现象 出现的原因可能是酰基对位上的取代基对酰基自由 
基稳定性影响较小, 该结论也与实验结果一致. 实验 中指出苯甲醛上的电子效应对反应的产率影响很小.

\subsection{2 亚胺自由基环化过程取代基电子效应}

为探究取代基电子效应对亚胺自由基环化过程 的影响, 我们模拟了酰基对位上含有取代基团、亚胺 自由基进攻位点对位含有取代基团以及氧基自由基 对位含有取代基团时亚胺自由基的环化反应，以同 样的算法和基组进行理论计算. 在之前优化的过渡 态结构基础上, 分别优化得到含有取代基反应的中 间体和过渡态结构, 整理亚胺自由基环化过程的活 化能数值, 以各取代基的取代基常数 $\sigma$ 为横坐标, 以 $\log k_{\mathrm{X}} / k_{\mathrm{H}}$ 为纵坐标, 根据取代基所在位置的不同分别 绘制 Hammett 曲线, 其结果如图 7 所示.

分析 Hammett 曲线, 对于亚胺自由基环化过程, 当取代基团在酰基对位时, 所得的回归直线如图 7(a) 所示, 直线拟合优度 $R^{2}$ 为 0.89 , 表明直线的拟合性很 好, 数据具有很好的线性相关性. 此外, 回归直线的 斜率为 -0.55 , 即反应常数 $\rho<0$, 也就是说取代基的取 代基常数 $\sigma$ 数值越小, 反应速率越快, 所以酰基对位 上连有吸电子基团(硝基、三氟甲基、氯原子)时不利 于环化过程的发生, 而酰基对位上连有给电子基团 (甲基、甲氧基、二甲基胺基)时有助于环化过程的发 生. 这是因为酰基对位的给电子基团会增大氰基上 的电荷密度, 这不仅有利于稳定亚胺自由基, 也有助 于亚胺自由基对苯环的亲核进攻，从而减小环化过 程的活化能. 然而, 当酰基对位被吸电子基团取代时, 氧基上的电荷密度会被降低, 从而不利于亚胺自由 基的亲核进攻.

当亚胺自由基进攻位点对位含有取代基团时, 得到的 Hammett 曲线如图 7(b)所示, 直线拟合优度 $R^{2}$ 为 0.15 , 这表明直线的拟合性很差, 即这组数据的 线性相关性很差. 因此, 亚胺自由基进攻位点对位上 的取代基电子效应对亚胺自由基环化过程的影响很 小. 当取代基在氰基自由基对位时, 所得 Hammett 曲 线如图 7(c) 所示, 直线拟合优度 $R^{2}$ 仅为 0.0001 , 直线 的拟合性非常差, 表明数据没有线性相关性. 因此, 当取代基在氰基对位时, 取代基效应影响很小.

\section{4 结论}

本文运用密度泛函理论计算, 系统研究了碱促
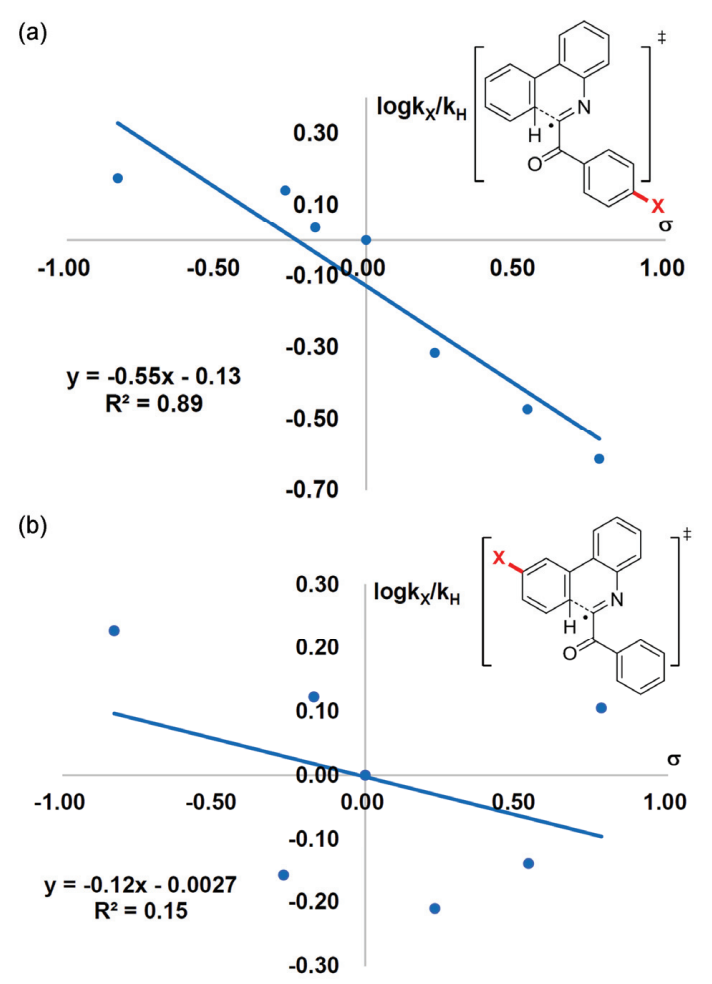

(c)



图 7 亚胺自由基环加成过程中不同取代位置的 Hammett 曲线. (a) 取代基- $X$ 在酰基对位; (b) 取代基- $\mathrm{X}$ 在苯环对位; (c) 取代基- $\mathrm{X}$ 在氰基对位. 其中取代基 $-\mathrm{X}$ 分别为; $-\mathrm{NMe}_{2}$ $(\sigma=-0.83) 、-\mathrm{OMe}(\sigma=-0.27) 、-\mathrm{Me}(\sigma=-0.17) 、-\mathrm{H}(\sigma=0)$ 、 $-\mathrm{Cl}(\sigma=0.23),-\mathrm{CF}_{3}(\sigma=0.54),-\mathrm{NO}_{2}(\sigma=0.78)$

均裂芳香取代合成 6-酰基菲啶的反应机理及不同位 置取代基电子效应. 计算结果表明, 反应首先进行的 是叔丁氧自由基和苯甲醛间自由基传递反应, 生成 酰基自由基, 之后酰基自由基进攻 2-氰基联苯上的 氧基形成亚胺自由基中间体. 亚胺自由基进攻相邻 芳环发生分子内环化, 最后由体系中的氧化剂过氧 叔丁醇氧化得到菲啶产物. 
此外，本文还运用 Hammett 曲线分别研究了酰 基自由基进攻氰基过程和亚胺自由基环化过程不同 位置上取代基的电子效应. 在酰基自由基进攻氧基 过程中, 氧基所在芳环对位上被吸电子基团取代时 有利于反应的进行，被给电子基团取代时不利于反
应的进行. 其他位置上的取代基对反应影响很小. 对 于亚胺自由基环化过程，酰基对位上的给电子基团 有助于自由基环化的发生, 而吸电子基团对环化过 程不利. 我们希望通过对不同位置取代基效应的研 究, 对实验提供指导.

\section{补充材料}

本文的补充材料见网络版 chem.scichina.com. 补充材料为作者提供的原始数据, 作者对其学术质量和内容负责.

\section{参考文献}

1 Bernardo PH, Wan KF, Sivaraman T, Xu J, Moore FK, Hung AW, Mok HYK, Yu VC, Chai CLL. J Med Chem, 2008, 51: 6699-6710

2 Zhu S, Ruchelman AL, Zhou N, Liu AA, Liu LF, LaVoie EJ. Bioorg Med Chem, 2005, 13: 6782-6794

3 Lynch MA, Duval O, Sukhanova A, Devy J, MacKay SP, Waigh RD, Nabiev I. Bioorg Med Chem Lett, 2001, 11: 2643-2646

4 Lewis WG, Green LG, Grynszpan F, Radić Z, Carlier PR, Taylor P, Finn MG, Sharpless KB. Angew Chem, 2002, 114: 1095-1099

5 Krane BD, Fagbule MO, Shamma M, Gözler B. J Nat Prod, 1984, 47: 1-43

6 Viladomat F, Sellés M, Cordina C, Bastida J. Planta Med, 1997, 63: 583

7 Ali AA, El Saved HM, Abdalliah OM, Steglich W. Phytochemistry, 1986, 25: 2399-2401

8 Stevens N, O'Connor N, Vishwasrao H, Samaroo D, Kandel ER, Akins DL, Drain CM, Turro NJ. J Am Chem Soc, 2008, 130: 7182-7183

9 Bondarev SL, Knyukshto VN, Tikhomirov SA, Pyrko AN. Opt Spectrosc, 2006, 100: 386-393

10 Zhang J, Lakowicz JR. J Phys Chem B, 2005, 109: 8701-8706

11 Simeon S, Rios JL, Villar A. Pharmazie, 1989, 44: 593-597

12 Phillips SD, Castle RN. J Heterocycl Chem, 1981, 18: 223-232

13 Abdel-Halim OB, Morikawa T, Ando S, Matsuda H, Yoshikawa M. J Nat Prod, 2004, 67: 1119-1124

14 Sripada L, Teske JA, Deiters A. Org Biomol Chem, 2008, 6: 263-265

15 Han YS, Kim SD, Kwon Y, Choi KH, Park LS. Mol Cryst Liq Cryst, 2006, 459: 119-128

16 Zhang L, Ang GY, Chiba S. Org Lett, 2010, 12: 3682-3685

17 Deb I, Yoshikai N. Org Lett, 2013, 15: 4254-4257

18 Wang WY, Feng X, Hu BL, Deng CL, Zhang XG. J Org Chem, 2013, 78: 6025-6030

19 Gerfaud T, Neuville L, Zhu J. Angew Chem Int Ed, 2009, 48: 572-577

20 Alonso R, Campos PJ, Rodríguez MA, Sampedro D. J Org Chem, 2008, 73: 2234-2239

21 Linsenmeier AM, Williams CM, Bräse S. J Org Chem, 2011, 76: 9127-9132

22 Alonso R, Campos PJ, García B, Rodríguez MA. Org Lett, 2006, 8: 3521-3523

23 Shou WG, Yang YY, Wang YG. J Org Chem, 2006, 71: 9241-9243

24 Pawlas J, Begtrup M. Org Lett, 2002, 4: 2687-2690

25 Shabashov D, Daugulis O. J Org Chem, 2007, 72: 7720-7725

26 Moreno I, Tellitu I, Etayo J, SanMartín R, Domínguez E. Tetrahedron, 2001, 57: 5403-5411

27 Tobisu M, Koh K, Furukawa T, Chatani N. Angew Chem Int Ed, 2012, 51: 11363-11366

28 Zhang B, Mück-Lichtenfeld C, Daniliuc CG, Studer A. Angew Chem Int Ed, 2013, 52: 10792-10795

29 Wang Q, Dong X, Xiao T, Zhou L. Org Lett, 2013, 15: 4846-4849

30 Li Z, Fan F, Yang J, Liu ZQ. Org Lett, 2014, 16: 3396-3399

31 Janza B, Studer A. Org Lett, 2006, 8: 1875-1878

32 Leifert D, Daniliuc CG, Studer A. Org Lett, 2013, 15: 6286-6289

33 Frisch MJ, Trucks GW, Schlegel HB, et al. Gaussian 09. Revision D 01. Wallingford CT: Gaussian Inc., 2013

34 Lee C, Yang W, Parr RG. Phys Rev B, 1988, 37: 785

35 Becke AD. J Chem Phys, 1993, 98: 5648-5652

36 Stephens PJ, Devlin FJ, Chabalowski CF, Frisch MJ. J Phys Chem, 1994, 98: 11623-11627

37 Adamo C, Barone V. J Chem Phys, 1999, 110: 6158-6170 


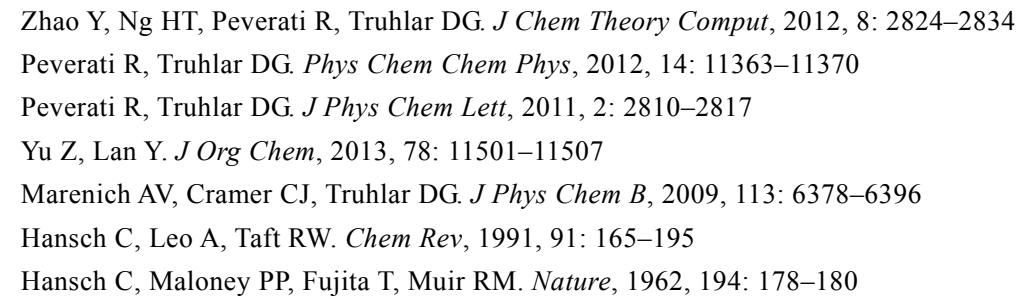

\title{
Mechanism and electronic effect for the synthesis of 6-aroylated phenanthridines: a theoretical study
}

\author{
Ting Xu, Xiaotian Qi, Yu Lan* \\ School of Chemistry and Chemical Engineering, Chongqing University, Chongqing 400030, China \\ *Corresponding author (email: lanyu@cqu.edu.cn)
}

\begin{abstract}
Density functional theory (DFT) calculations were employed to study the mechanism for the synthesis of 6-aroylated phenanthridines via base promoted homolytic aromatic substitution. Computational results suggest that the radical transfer process between butyloxycarbonyl radical and benzaldehyde is the rate-determining step. Subsequent nucleophilic addition of acyl radical towards cyano group and the radical cyclization step are facile process. Besides, the electronic effect of substituents on different sites was also investigated using the Hammett plot. The obtained results indicate that, the electron-withdrawing group on para-position of cyano group would facilitates the nucleophilic addition of acyl radical, and the electron-donating group on para-position of acyl group would promote the radical cyclization step.
\end{abstract}

Keywords: phenanthridines, DFT calculation, free radical reaction, Hammett plot, the electronic effect of substituents

doi: $10.1360 / \mathrm{N} 032015-00217$ 